\title{
Sorting by Exchanging
}

\author{
Grzegorz Bancerek \\ Białystok Technical University \\ Poland
}

\begin{abstract}
Summary. We show that exchanging of pairs in an array which are in incorrect order leads to sorted array. It justifies correctness of Bubble Sort, Insertion Sort, and Quicksort.
\end{abstract}

MML identifier: EXCHSORT, version: $\underline{7.11 .07 \quad 4.156 .1112}$

The notation and terminology used here have been introduced in the following papers: [20], [6], [11], [1], [8], [16], [12], [13], [10], [9], [17], [18], [3], [4], [2], [7], [14], [21], [22], [19], [5], and [15].

\section{Preliminaries}

We adopt the following convention: $\alpha, \beta, \gamma, \delta$ denote ordinal numbers, $k$ denotes a natural number, and $x, y, z, t, X, Y, Z$ denote sets.

The following propositions are true:

(1) $x \in(\alpha+\beta) \backslash \alpha$ iff there exists $\gamma$ such that $x=\alpha+\gamma$ and $\gamma \in \beta$.

(2) Suppose $\alpha \in \beta$ and $\gamma \in \delta$. Then $\gamma \neq \alpha$ and $\gamma \neq \beta$ and $\delta \neq \alpha$ and $\delta \neq \beta$ or $\gamma \in \alpha$ and $\delta=\alpha$ or $\gamma \in \alpha$ and $\delta=\beta$ or $\gamma=\alpha$ and $\delta \in \beta$ or $\gamma=\alpha$ and $\delta=\beta$ or $\gamma=\alpha$ and $\beta \in \delta$ or $\alpha \in \gamma$ and $\delta=\beta$ or $\gamma=\beta$ and $\beta \in \delta$.

(3) If $x \notin y$, then $(y \cup\{x\}) \backslash y=\{x\}$.

(4) $\operatorname{succ} x \backslash x=\{x\}$.

(5) Let $f$ be a function, $r$ be a binary relation, and given $x$. Then $x \in f^{\circ} r$ if and only if there exist $y, z$ such that $\langle y, z\rangle \in r$ and $\langle y, z\rangle \in \operatorname{dom} f$ and $f(y, z)=x$.

(6) If $\alpha \backslash \beta \neq \emptyset$, then $\inf (\alpha \backslash \beta)=\beta$ and $\sup (\alpha \backslash \beta)=\alpha$ and $\bigcup(\alpha \backslash \beta)=\bigcup \alpha$. 
(7) If $\alpha \backslash \beta$ is non empty and finite, then there exists a natural number $n$ such that $\alpha=\beta+n$.

\section{ARRAYS}

Let $f$ be a set. We say that $f$ is segmental if and only if:

(Def. 1) There exist $\alpha, \beta$ such that $\pi_{1}(f)=\alpha \backslash \beta$.

In the sequel $f, g$ denote functions.

The following two propositions are true:

(8) If $\operatorname{dom} f=\operatorname{dom} g$ and $f$ is segmental, then $g$ is segmental.

(9) If $f$ is segmental, then for all $\alpha, \beta, \gamma$ such that $\alpha \subseteq \beta \subseteq \gamma$ and $\alpha$, $\gamma \in \operatorname{dom} f$ holds $\beta \in \operatorname{dom} f$.

Let us observe that every function which is transfinite sequence-like is also segmental and every function which is finite sequence-like is also segmental.

Let us consider $\alpha$ and let $s$ be a set. We say that $s$ is $\alpha$-based if and only if: (Def. 2) If $\beta \in \pi_{1}(s)$, then $\alpha \in \pi_{1}(s)$ and $\alpha \subseteq \beta$.

We say that $s$ is $\alpha$-limited if and only if:

(Def. 3) $\quad \alpha=\sup \pi_{1}(s)$.

Next we state two propositions:

(10) $f$ is $\alpha$-based and segmental iff there exists $\beta$ such that $\operatorname{dom} f=\beta \backslash \alpha$ and $\alpha \subseteq \beta$.

(11) $f$ is $\beta$-limited, non empty, and segmental iff there exists $\alpha$ such that $\operatorname{dom} f=\beta \backslash \alpha$ and $\alpha \in \beta$.

Let us observe that every function which is transfinite sequence-like is also 0-based and every function which is finite sequence-like is also 1-based.

The following three propositions are true:

(12) $f$ is inf dom $f$-based.

(13) $f$ is $\sup \operatorname{dom} f$-limited.

(14) If $f$ is $\beta$-limited and $\alpha \in \operatorname{dom} f$, then $\alpha \in \beta$.

Let us consider $f$. The functor base $f$ yielding an ordinal number is defined as follows:

(Def. 4)(i) $f$ is base $f$-based if there exists $\alpha$ such that $\alpha \in \operatorname{dom} f$,

(ii) base $f=0$, otherwise.

The functor limit $f$ yields an ordinal number and is defined as follows:

(Def. 5)(i) $\quad f$ is limit $f$-limited if there exists $\alpha$ such that $\alpha \in \operatorname{dom} f$,

(ii) $\operatorname{limit} f=0$, otherwise.

Let us consider $f$. The functor length $f$ yielding an ordinal number is defined as follows: 
(Def. 6) length $f=\operatorname{limit} f-$ base $f$.

We now state four propositions:

(15) base $\emptyset=0$ and limit $\emptyset=0$ and length $\emptyset=0$.

(16) $\operatorname{limit} f=\sup \operatorname{dom} f$.

(17) $f$ is limit $f$-limited.

(18) Every empty set is $\alpha$-based.

Let us consider $\alpha, X, Y$. Note that there exists a transfinite sequence which is $Y$-defined, $X$-valued, $\alpha$-based, segmental, finite, and empty.

An array is a segmental function.

Let $A$ be an array. Observe that $\operatorname{dom} A$ is ordinal-membered.

We now state the proposition

(19) For every array $f$ holds $f$ is 0-limited iff $f$ is empty.

Let us mention that every array which is 0-based is also transfinite sequencelike.

Let us consider $X$. An array of $X$ is a $X$-valued array.

Let $X$ be a 1-sorted structure. An array of $X$ is an array of the carrier of $X$.

Let us consider $\alpha, X$. An array of $\alpha, X$ is a $\alpha$-defined array of $X$.

In the sequel $A, B, C$ denote arrays.

Next we state several propositions:

(20) base $f=\inf \operatorname{dom} f$.

(21) $f$ is base $f$-based.

(22) $\operatorname{dom} A=\operatorname{limit} A \backslash$ base $A$.

(23) If $\operatorname{dom} A=\alpha \backslash \beta$ and $A$ is non empty, then base $A=\beta$ and limit $A=\alpha$.

(24) For every transfinite sequence $f$ holds base $f=0$ and limit $f=\operatorname{dom} f$ and length $f=\operatorname{dom} f$.

Let us consider $\alpha, \beta, X$. Note that there exists an array of $\alpha, X$ which is $\beta$-based, natural-valued, integer-valued, real-valued, complex-valued, and finite.

Let us consider $\alpha, x$. Note that $\{\langle\alpha, x\rangle\}$ is segmental.

Let us consider $\alpha$ and let $x$ be a natural number. Observe that $\{\langle\alpha, x\rangle\}$ is natural-valued.

Let us consider $\alpha$ and let $x$ be a real number. One can verify that $\{\langle\alpha, x\rangle\}$ is real-valued.

Let us consider $\alpha$, let $X$ be a non empty set, and let $x$ be an element of $X$. One can check that $\{\langle\alpha, x\rangle\}$ is $X$-valued.

Let us consider $\alpha, x$. One can check that $\{\langle\alpha, x\rangle\}$ is $\alpha$-based and $\operatorname{succ} \alpha$ limited.

Let us consider $\beta$. Note that there exists an array which is non empty, $\beta$ based, natural-valued, integer-valued, real-valued, complex-valued, and finite. Let $X$ be a non empty set. Note that there exists an array which is non empty, $\beta$-based, finite, and $X$-valued. 
Let $s$ be a transfinite sequence. We introduce $s$ last as a synonym of last $s$.

Let $A$ be an array. The functor last $A$ is defined by:

(Def. 7) $\quad$ last $A=A(\bigcup \operatorname{dom} A)$.

\section{Descending Sequences}

Let $f$ be a function. We say that $f$ is descending if and only if:

(Def. 8) For all $\alpha, \beta$ such that $\alpha, \beta \in \operatorname{dom} f$ and $\alpha \in \beta$ holds $f(\beta) \subset f(\alpha)$.

We now state four propositions:

(25) For every finite array $f$ such that for every $\alpha$ such that $\alpha$, succ $\alpha \in \operatorname{dom} f$ holds $f(\operatorname{succ} \alpha) \subset f(\alpha)$ holds $f$ is descending.

(26) For every array $f$ such that length $f=\omega$ and for every $\alpha$ such that $\alpha$, succ $\alpha \in \operatorname{dom} f$ holds $f(\operatorname{succ} \alpha) \subset f(\alpha)$ holds $f$ is descending.

(27) For every transfinite sequence $f$ such that $f$ is descending and $f(0)$ is finite holds $f$ is finite.

(28) Let $f$ be a transfinite sequence. Suppose $f$ is descending and $f(0)$ is finite and for every $\alpha$ such that $f(\alpha) \neq \emptyset$ holds succ $\alpha \in \operatorname{dom} f$. Then last $f=\emptyset$.

The scheme $A$ deals with a transfinite sequence $\mathcal{A}$ and a unary functor $\mathcal{F}$ yielding a set, and states that:

$\mathcal{A}$ is finite

provided the parameters meet the following requirements:

- $\mathcal{F}(\mathcal{A}(0))$ is finite, and

- For every $\alpha$ such that succ $\alpha \in \operatorname{dom} \mathcal{A}$ and $\mathcal{F}(\mathcal{A}(\alpha))$ is finite holds $\mathcal{F}(\mathcal{A}(\operatorname{succ} \alpha)) \subset \mathcal{F}(\mathcal{A}(\alpha))$.

\section{SWAP}

Let us consider $X$, let $f$ be a $X$-defined function, and let $\alpha, \beta$ be sets. Note that $\operatorname{Swap}(f, \alpha, \beta)$ is $X$-defined.

Let $X$ be a set, let $f$ be a $X$-valued function, and let $x, y$ be sets. Note that $\operatorname{Swap}(f, x, y)$ is $X$-valued.

The following propositions are true:

(29) If $x, y \in \operatorname{dom} f$, then $(\operatorname{Swap}(f, x, y))(x)=f(y)$.

(30) For every array $f$ of $X$ such that $x, y \in \operatorname{dom} f$ holds $(\operatorname{Swap}(f, x, y))_{x}=$ $f_{y}$.

(31) If $x, y \in \operatorname{dom} f$, then $(\operatorname{Swap}(f, x, y))(y)=f(x)$.

(32) For every array $f$ of $X$ such that $x, y \in \operatorname{dom} f$ holds $(\operatorname{Swap}(f, x, y))_{y}=$ $f_{x}$. 
(33) If $z \neq x$ and $z \neq y$, then $(\operatorname{Swap}(f, x, y))(z)=f(z)$.

(34) For every array $f$ of $X$ such that $z \in \operatorname{dom} f$ and $z \neq x$ and $z \neq y$ holds $(\operatorname{Swap}(f, x, y))_{z}=f_{z}$.

(35) If $x, y \in \operatorname{dom} f$, then $\operatorname{Swap}(f, x, y)=\operatorname{Swap}(f, y, x)$.

Let $X$ be a non empty set. Observe that there exists a $X$-valued non empty function which is onto.

Let $X$ be a non empty set, let $f$ be an onto $X$-valued non empty function, and let $x, y$ be elements of $\operatorname{dom} f$. Note that $\operatorname{Swap}(f, x, y)$ is onto.

Let us consider $A$ and let us consider $x, y$. Note that $\operatorname{Swap}(A, x, y)$ is segmental.

We now state the proposition

(36) If $x, y \in \operatorname{dom} A$, then $\operatorname{Swap}(\operatorname{Swap}(A, x, y), x, y)=A$.

Let $A$ be a real-valued array and let us consider $x, y$. One can verify that $\operatorname{Swap}(A, x, y)$ is real-valued.

\section{Permutations}

Let $A$ be an array. An array is called a permutation of $A$ if:

(Def. 9) There exists a permutation $f$ of $\operatorname{dom} A$ such that it $=A \cdot f$.

We now state several propositions:

(37) For every permutation $B$ of $A$ holds $\operatorname{dom} B=\operatorname{dom} A$ and $\operatorname{rng} B=\operatorname{rng} A$.

(38) $A$ is a permutation of $A$.

(39) If $A$ is a permutation of $B$, then $B$ is a permutation of $A$.

(40) If $A$ is a permutation of $B$ and $B$ is a permutation of $C$, then $A$ is a permutation of $C$.

(41) $\operatorname{Swap}\left(\operatorname{id}_{X}, x, y\right)$ is one-to-one.

Let $X$ be a non empty set and let $x, y$ be elements of $X$.

Note that $\operatorname{Swap}\left(\operatorname{id}_{X}, x, y\right)$ is one-to-one, $X$-valued, and $X$-defined.

Let $X$ be a non empty set and let $x, y$ be elements of $X$.

Note that $\operatorname{Swap}\left(\operatorname{id}_{X}, x, y\right)$ is onto and total.

Let $X, Y$ be non empty sets, let $f$ be a function from $X$ into $Y$, and let $x$, $y$ be elements of $X$. Then $\operatorname{Swap}(f, x, y)$ is a function from $X$ into $Y$.

Next we state three propositions:

(42) If $x, y \in X$ and $f=\operatorname{Swap}\left(\operatorname{id}_{X}, x, y\right)$ and $X=\operatorname{dom} A$, then $\operatorname{Swap}(A, x, y)=A \cdot f$.

(43) If $x, y \in \operatorname{dom} A$, then $\operatorname{Swap}(A, x, y)$ is a permutation of $A$ and $A$ is a permutation of $\operatorname{Swap}(A, x, y)$.

(44) Suppose $x, y \in \operatorname{dom} A$ and $A$ is a permutation of $B$. Then $\operatorname{Swap}(A, x, y)$ is a permutation of $B$ and $A$ is a permutation of $\operatorname{Swap}(B, x, y)$. 


\section{EXCHANGING}

Let $O$ be a relational structure and let $A$ be an array of $O$. We say that $A$ is ascending if and only if:

(Def. 10) For all $\alpha, \beta$ such that $\alpha, \beta \in \operatorname{dom} A$ and $\alpha \in \beta$ holds $A_{\alpha} \leq A_{\beta}$.

The functor inversions $A$ is defined by:

(Def. 11) inversions $A=\{\langle\alpha, \beta\rangle ; \alpha$ ranges over elements of $\operatorname{dom} A, \beta$ ranges over elements of $\left.\operatorname{dom} A: \alpha \in \beta \wedge A_{\alpha} \not \leq A_{\beta}\right\}$.

Let $O$ be a relational structure. One can verify that every empty array of $O$ is ascending. Let $A$ be an empty array of $O$. One can verify that inversions $A$ is empty.

In the sequel $O$ is a connected non empty poset and $R, Q$ are arrays of $O$.

We now state the proposition

(45) For every $O$ and for all elements $x, y$ of $O$ holds $x>y$ iff $x \not \leq y$.

Let us consider $O, R$. Then inversions $R$ can be characterized by the condition:

(Def. 12) inversions $R=\{\langle\alpha, \beta\rangle ; \alpha$ ranges over elements of $\operatorname{dom} R, \beta$ ranges over elements of $\left.\operatorname{dom} R: \alpha \in \beta \wedge R_{\alpha}>R_{\beta}\right\}$.

Next we state two propositions:

(46) $\langle x, y\rangle \in$ inversions $R$ iff $x, y \in \operatorname{dom} R$ and $x \in y$ and $R_{x}>R_{y}$.

(47) inversions $R \subseteq \operatorname{dom} R \times \operatorname{dom} R$.

Let us consider $O$ and let $R$ be a finite array of $O$. Observe that inversions $R$ is finite.

We now state three propositions:

(48) $\quad R$ is ascending iff inversions $R=\emptyset$.

(49) If $\langle x, y\rangle \in$ inversions $R$, then $\langle y, x\rangle \notin$ inversions $R$.

(50) If $\langle x, y\rangle,\langle y, z\rangle \in$ inversions $R$, then $\langle x, z\rangle \in$ inversions $R$.

Let us consider $O, R$. Note that inversions $R$ is relation-like.

Let us consider $O, R$. Note that inversions $R$ is asymmetric and transitive.

Let us consider $O$ and let $\alpha, \beta$ be elements of $O$. Let us note that the predicate $\alpha<\beta$ is antisymmetric.

Next we state several propositions:

(51) If $\langle x, y\rangle \in$ inversions $R$, then $\langle x, y\rangle \notin$ inversions $\operatorname{Swap}(R, x, y)$.

(52) If $x, y \in \operatorname{dom} R$ and $z \neq x$ and $z \neq y$ and $t \neq x$ and $t \neq y$, then $\langle z$, $t\rangle \in$ inversions $R$ iff $\langle z, t\rangle \in$ inversions $\operatorname{Swap}(R, x, y)$.

(53) If $\langle x, y\rangle \in$ inversions $R$, then $\langle z, y\rangle \in$ inversions $R$ and $z \in x$ iff $\langle z$, $x\rangle \in$ inversions $\operatorname{Swap}(R, x, y)$.

(54) If $\langle x, y\rangle \in$ inversions $R$, then $\langle z, x\rangle \in \operatorname{inversions~} R$ iff $z \in x$ and $\langle z$, $y\rangle \in$ inversions $\operatorname{Swap}(R, x, y)$. 
(55) If $\langle x, y\rangle \in$ inversions $R$ and $z \in y$ and $\langle x, z\rangle \in$ inversions $\operatorname{Swap}(R, x, y)$, then $\langle x, z\rangle \in$ inversions $R$.

(56) If $\langle x, y\rangle \in$ inversions $R$ and $x \in z$ and $\langle z, y\rangle \in$ inversions $\operatorname{Swap}(R, x, y)$, then $\langle z, y\rangle \in$ inversions $R$.

(57) If $\langle x, y\rangle \in$ inversions $R$ and $y \in z$ and $\langle x, z\rangle \in$ inversions $\operatorname{Swap}(R, x, y)$, then $\langle y, z\rangle \in$ inversions $R$.

(58) If $\langle x, y\rangle \in$ inversions $R$, then $y \in z$ and $\langle x, z\rangle \in$ inversions $R$ iff $\langle y$, $z\rangle \in$ inversions $\operatorname{Swap}(R, x, y)$.

Let us consider $O, R, x, y$. The functor $\subseteq_{x, y}^{R}$ yields a function and is defined by:

(Def. 13) $\subseteq_{x, y}^{R}=\operatorname{Swap}\left(\operatorname{id}_{\operatorname{dom} R}, x, y\right) \times \operatorname{Swap}\left(\operatorname{id}_{\operatorname{dom} R}, x, y\right)+\cdot \operatorname{id}_{\{x\} \times(\operatorname{succ} y \backslash x) \cup(\operatorname{succ} y \backslash x) \times\{y\}}$.

Next we state the proposition

(59) $\gamma \in \operatorname{succ} \beta \backslash \alpha$ iff $\alpha \subseteq \gamma \subseteq \beta$.

We adopt the following convention: $T$ is a non empty array of $O$ and $p, q$, $r, s$ are elements of $\operatorname{dom} T$.

The following propositions are true:

(60) $\operatorname{succ} q \backslash p \subseteq \operatorname{dom} T$.

(61) $\operatorname{dom} \subseteq_{p, q}^{T}=\operatorname{dom} T \times \operatorname{dom} T$ and $\operatorname{rng} \subseteq_{p, q}^{T} \subseteq \operatorname{dom} T \times \operatorname{dom} T$.

(62) If $p \subseteq r \subseteq q$, then $\left(\subseteq_{p, q}^{T}\right)(p, r)=\langle p, r\rangle$ and $\left(\subseteq_{p, q}^{T}\right)(r, q)=\langle r, q\rangle$.

(63) If $r \neq p$ and $s \neq q$ and $f=\operatorname{Swap}\left(\operatorname{id}_{\operatorname{dom} T}, p, q\right)$, then $\left(\subseteq_{p, q}^{T}\right)(r, s)=\langle f(r)$, $f(s)\rangle$.

(64) If $r \in p$ and $f=\operatorname{Swap}\left(\operatorname{id}_{\operatorname{dom} T}, p, q\right)$, then $\left(\subseteq_{p, q}^{T}\right)(r, q)=\langle f(r), f(q)\rangle$ and $\left(\subseteq_{p, q}^{T}\right)(r, p)=\langle f(r), f(p)\rangle$

(65) If $q \in r$ and $f=\operatorname{Swap}\left(\operatorname{id}_{\operatorname{dom} T}, p, q\right)$, then $\left(\subseteq_{p, q}^{T}\right)(p, r)=\langle f(p), f(r)\rangle$ and $\left(\subseteq_{p, q}^{T}\right)(q, r)=\langle f(q), f(r)\rangle$.

(66) If $p \in q$, then $\left(\subseteq_{p, q}^{T}\right)(p, q)=\langle p, q\rangle$.

(67) If $p \in q$ and $r \neq p$ and $r \neq q$ and $s \neq p$ and $s \neq q$, then $\left(\subseteq_{p, q}^{T}\right)(r, s)=\langle r$, $s\rangle$.

(68) If $r \in p$ and $p \in q$, then $\left(\subseteq_{p, q}^{T}\right)(r, p)=\langle r, q\rangle$ and $\left(\subseteq_{p, q}^{T}\right)(r, q)=\langle r, p\rangle$.

(69) If $p \in s$ and $s \in q$, then $\left(\subseteq_{p, q}^{T}\right)(p, s)=\langle p, s\rangle$ and $\left(\subseteq_{p, q}^{T}\right)(s, q)=\langle s, q\rangle$.

(70) If $p \in q$ and $q \in s$, then $\left(\subseteq_{p, q}^{T}\right)(p, s)=\langle q, s\rangle$ and $\left(\subseteq_{p, q}^{T}\right)(q, s)=\langle p, s\rangle$.

(71) If $p \in q$, then $\subseteq_{p, q}^{T}$ 「(inversions $\operatorname{Swap}(T, p, q)$ qua set) is one-to-one.

Let us consider $O, R, x, y, z$. Note that $\left(\subseteq_{x, y}^{R}\right)^{\circ} z$ is relation-like. 


\section{Correctness of Sorting By Exchanging}

The following proposition is true

(72) If $\langle x, y\rangle \in$ inversions $R$, then $\left(\subseteq_{x, y}^{R}\right)^{\circ}$ inversions $\operatorname{Swap}(R, x, y) \subset$ inversions $R$.

Let $R$ be a finite function and let us consider $x, y$. One can check that $\operatorname{Swap}(R, x, y)$ is finite.

Next we state two propositions:

(73) For every array $R$ of $O$ such that $\langle x, y\rangle \in$ inversions $R$ and inversions $R$

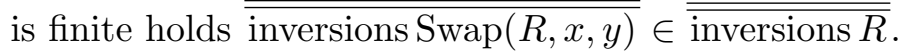

(74) For every finite array $R$ of $O$ such that $\langle x, y\rangle \in$ inversions $R$ holds $\overline{\overline{\text { inversions Swap }(R, x, y)}}<\overline{\overline{\text { inversions } R}}$.

Let us consider $O, R$. A non empty array is called a computation of $R$ if it satisfies the conditions (Def. 14).

(Def. 14)(i) $\quad$ It(base it) $=R$,

(ii) for every $\alpha$ such that $\alpha \in \operatorname{dom}$ it holds it $(\alpha)$ is an array of $O$, and

(iii) for every $\alpha$ such that $\alpha$, succ $\alpha \in$ dom it there exist $R, x, y$ such that $\langle x, y\rangle \in$ inversions $R$ and $\operatorname{it}(\alpha)=R$ and $\operatorname{it}(\operatorname{succ} \alpha)=\operatorname{Swap}(R, x, y)$.

We now state the proposition

(75) $\{\langle\alpha, R\rangle\}$ is a computation of $R$.

Let us consider $O, R, \alpha$. One can check that there exists a computation of $R$ which is $\alpha$-based and finite.

Let us consider $O, R$, let $C$ be a computation of $R$, and let us consider $x$. One can check that $C(x)$ is segmental, function-like, and relation-like.

Let us consider $O, R$, let $C$ be a computation of $R$, and let us consider $x$. Observe that $C(x)$ is the carrier of $O$-valued.

Let us consider $O, R$ and let $C$ be a computation of $R$. Observe that last $C$ is segmental, relation-like, and function-like.

Let us consider $O, R$ and let $C$ be a computation of $R$. Observe that last $C$ is the carrier of $O$-valued.

Let us consider $O, R$ and let $C$ be a computation of $R$. We say that $C$ is complete if and only if:

(Def. 15) last $C$ is ascending.

One can prove the following three propositions:

(76) For every 0-based computation $C$ of $R$ such that $R$ is a finite array of $O$ holds $C$ is finite.

(77) Let $C$ be a 0-based computation of $R$. Suppose $R$ is a finite array of $O$ and for every $\alpha$ such that inversions $C(\alpha) \neq \emptyset$ holds succ $\alpha \in \operatorname{dom} C$. Then $C$ is complete. 
(78) Let $C$ be a finite computation of $R$. Then last $C$ is a permutation of $R$ and for every $\alpha$ such that $\alpha \in \operatorname{dom} C$ holds $C(\alpha)$ is a permutation of $R$.

\section{Existence of Complete Computations}

Next we state three propositions:

(79) For every 0-based finite array $A$ of $X$ such that $A \neq \emptyset$ holds last $A \in X$.

(80) $\operatorname{last}\langle x\rangle=x$.

(81) For every 0-based finite array $A$ holds last $\left(A^{\frown}\langle x\rangle\right)=x$.

Let $X$ be a set. Observe that every element of $X^{\omega}$ is $X$-valued.

The scheme $A$ deals with a unary functor $\mathcal{F}$ yielding a set, a non empty set $\mathcal{A}$, a set $\mathcal{B}$, and a binary predicate $\mathcal{P}$, and states that:

There exists a finite 0-based non empty array $f$ and there exists an element $k$ of $\mathcal{A}$ such that

(i) $\quad k=$ last $f$,

(ii) $\mathcal{F}(k)=\emptyset$,

(iii) $\quad f(0)=\mathcal{B}$, and

(iv) for every $\alpha$ such that $\operatorname{succ} \alpha \in \operatorname{dom} f$ there exist elements

$x, y$ of $\mathcal{A}$ such that $x=f(\alpha)$ and $y=f(\operatorname{succ} \alpha)$ and $\mathcal{P}[x, y]$

provided the following requirements are met:

- $\mathcal{B} \in \mathcal{A}$,

- $\mathcal{F}(\mathcal{B})$ is finite, and

- For every element $x$ of $\mathcal{A}$ such that $\mathcal{F}(x) \neq \emptyset$ there exists an element $y$ of $\mathcal{A}$ such that $\mathcal{P}[x, y]$ and $\mathcal{F}(y) \subset \mathcal{F}(x)$.

In the sequel $A$ is an array and $B$ is a permutation of $A$.

We now state the proposition

(82) $B \in(\operatorname{rng} A)^{\operatorname{dom} A}$.

Let $A$ be a real-valued array. One can verify that every permutation of $A$ is real-valued.

Let us consider $\alpha$ and let $X$ be a non empty set. Observe that every element of $X^{\alpha}$ is transfinite sequence-like.

Let us consider $X$ and let $Y$ be a real-membered non empty set. One can check that every element of $Y^{X}$ is real-valued.

Let us consider $X$ and let $A$ be an array of $X$. One can check that every permutation of $A$ is $X$-valued.

Let $X$ be a set, let $Z$ be a set, and let $Y$ be a subset of $Z$. Note that every element of $Y^{X}$ is $Z$-valued.

One can prove the following propositions:

(83) Every $X$-defined $Y$-valued binary relation is a relation between $X$ and $Y$. 
(84) For every finite ordinal number $\alpha$ and for every $x$ such that $x \in \alpha$ holds $x=0$ or there exists $\beta$ such that $x=\operatorname{succ} \beta$.

(85) For every 0-based finite non empty array $A$ of $O$ holds there exists a 0-based computation of $A$ which is complete.

(86) For every 0-based finite non empty array $A$ of $O$ holds there exists a permutation of $A$ which is ascending.

Let us consider $O$ and let $A$ be a 0-based finite array of $O$. Observe that there exists a permutation of $A$ which is ascending.

\section{REFERENCES}

[1] Grzegorz Bancerek. Cardinal numbers. Formalized Mathematics, 1(2):377-382, 1990.

[2] Grzegorz Bancerek. Ordinal arithmetics. Formalized Mathematics, 1(3):515-519, 1990.

[3] Grzegorz Bancerek. The ordinal numbers. Formalized Mathematics, 1(1):91-96, 1990.

[4] Grzegorz Bancerek. Sequences of ordinal numbers. Formalized Mathematics, 1(2):281290, 1990.

[5] Grzegorz Bancerek. Directed sets, nets, ideals, filters, and maps. Formalized Mathematics, 6(1):93-107, 1997.

[6] Grzegorz Bancerek. Mizar analysis of algorithms: Preliminaries. Formalized Mathematics, 15(3):87-110, 2007, doi:10.2478/v10037-007-0011-x.

[7] Grzegorz Bancerek. Veblen hierarchy. Formalized Mathematics, 19(2):83-92, 2011, doi: 10.2478/v10037-011-0014-5.

[8] Grzegorz Bancerek and Krzysztof Hryniewiecki. Segments of natural numbers and finite sequences. Formalized Mathematics, 1(1):107-114, 1990.

[9] Grzegorz Bancerek and Andrzej Trybulec. Miscellaneous facts about functions. Formalized Mathematics, 5(4):485-492, 1996.

[10] Czesław Byliński. Basic functions and operations on functions. Formalized Mathematics, 1(1):245-254, 1990.

[11] Czesław Byliński. Binary operations. Formalized Mathematics, 1(1):175-180, 1990.

[12] Czesław Byliński. Functions and their basic properties. Formalized Mathematics, 1(1):5565, 1990.

[13] Czesław Byliński. Functions from a set to a set. Formalized Mathematics, 1(1):153-164, 1990.

[14] Czesław Byliński. Partial functions. Formalized Mathematics, 1(2):357-367, 1990.

[15] Czesław Byliński. Some basic properties of sets. Formalized Mathematics, 1(1):47-53, 1990.

[16] Agata Darmochwał. Finite sets. Formalized Mathematics, 1(1):165-167, 1990

[17] Andrzej Trybulec. On the sets inhabited by numbers. Formalized Mathematics, 11(4):341$347,2003$.

[18] Wojciech A. Trybulec and Grzegorz Bancerek. Kuratowski - Zorn lemma. Formalized Mathematics, 1(2):387-393, 1990.

[19] Zinaida Trybulec. Properties of subsets. Formalized Mathematics, 1(1):67-71, 1990.

[20] Tetsuya Tsunetou, Grzegorz Bancerek, and Yatsuka Nakamura. Zero-based finite sequences. Formalized Mathematics, 9(4):825-829, 2001.

[21] Edmund Woronowicz. Relations and their basic properties. Formalized Mathematics, 1(1):73-83, 1990.

[22] Edmund Woronowicz and Anna Zalewska. Properties of binary relations. Formalized Mathematics, 1(1):85-89, 1990. 